\title{
Videos docentes para estudiantes del Grado en Matemáticas
}

\author{
Beatriz Estrada López \\ Departamento de Matemáticas Fundamentales \\ Facultad de Ciencias \\ Universidad Nacional de Educación a Distancia (UNED) \\ Paseo Senda de Rey, 9. 28040 Madrid. Spain. \\ bestra@mat.uned.es \\ Presentado en eXIDO16 (2016)
}

\section{Introducción:}

En mi larga experiencia docente en la educación a distancia he experimentado con distintos tipos de materiales que puedan resultar de ayuda en el aprendizaje de las matemáticas, especialmente cuando el mayor esfuerzo del aprendizaje recae en el trabajo autónomo del estudiante, sobre la base de un buen libro de texto y otros materiales de apoyo. Los estudiantes buscan cada vez más recursos audiovisuales en internet y se encuentran, entre otras, con la dificultad de decidir sobre su validez (contenidos y calidad). Se encuentran gran cantidad de vídeos con orientación práctica, es decir, vídeos en los que se resuelven ejercicios de matemáticas. Pero es totalmente inusual encontrar vídeos con contenidos matemáticos más teóricos. Vídeos de matemáticas para matemáticos.

El objetivo de la comunicación es presentar mi experiencia, y evolución, en la elaboración de vídeos dirigidos específicamente a estudiantes de primer curso del Grado en Matemáticas de la UNED. Elaboración que ha estado guiada por la detección de las carencias más importantes que aparecen en el salto entre estudios preuniversitarios y universitarios, y por la demanda de los propios estudiantes. Dichas carencias, se acentúan en la enseñanza a distancia (o semipresencial, como es el caso de la UNED donde imparto mi docencia) aunque se amortiguan, parcialmente, por sel nivel de madurez y compromiso de los estudiantes.

La principal de estas carencias es la falta de familiaridad con el uso formal del lenguaje lógicomatemático, que les hace muy difícil la lectura y comprensión de un libro de texto de matemáticas.

\section{El modelo docente de la UNED}

En el modelo de enseñanza semipresencial de la UNED todos los estudiantes (de España y el extranjero) con independencia de la localidad en la que se hayan matriculado tienen acceso a un curso virtual por cada asignatura. En este espacio comparten recursos (materiales impresos, vídeos, etc.) y espacios de trabajo y comunicación (foros temáticos, videocenferencias, etc). Todos los estudiantes tienen comunicación directa el Equipo Docente (profesor(es)) responsable de la asignatura. Además, disponen de clases de 1 hora (aprox.) de apoyo semanal en su centro local impartidas por tutores.

El curso virtual es el espacio común en el que todos los estudiantes, que trabajan sobre un mismo libro de texto, resuelven sus dudas, practican con ejercicios adicionales y acceden a todos los recursos de apoyo. Este es el espacio ideal para hacer llegar de modo organizado distintos tipos de vídeos docentes a los estudiantes. Tanto vídeos elaborados con distintas herramientas que se 
puedan encontrar en el mercado (software libre o no) como sesiones de clase en línea, ya sean en directo o en diferido.

La UNED desarrolló su propia plataforma de vídeo y webconferencia, la plataforma AVIP (audio y vídeo sobre IP) que incluye también repositorios de vídeos (Cadena Campus) de libre acceso. La herramienta para la realización de webconferencias está disponible en los cursos virtuales y permite realizar clases en línea invitando a todos los estudiantes de una asignatura y (opcionalmente) grabar la sesión, para que la puedan visualizar los que no pudieron asistir en directo. Los estudiantes conectados pueden hacer preguntas al profesor ponente a través de un chat o bien pedir la palabra e intervenir con voz y/o imagen.

Esta tecnología comenzó a utilizarse de forma sistemática para la docencia en curos virtuales desde la implantación de los nuevos estudios de grado en el curso 2010/11. Bien para la realización de grabaciones sesiones de webconferencia en directo o diferido, o bien alojando vídeos elaborados con otro tipo de software en los repositorios.

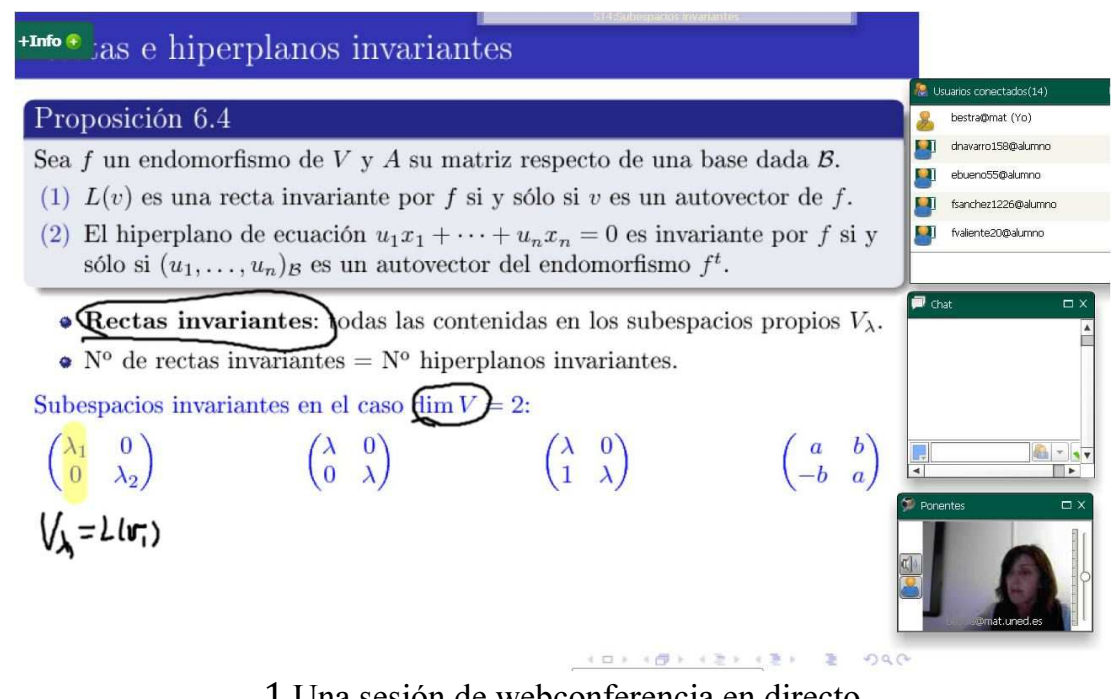

1 Una sesión de webconferencia en directo

\section{Tipos de vídeos por contenido y duración}

Los primeros años experimenté con vídeos de dos tipos:

- Lo que podríamos llamar mini-vídeos (5-6 min.) o vídeos cortos con una orientación fundamentalmente práctica, dirigida a la resolución de ejercicios fundamentalmente o aclarar alguna idea o error reiterado.

- Vídeos largos de una hora aproximada de duración, como resultado de la grabación de clases impartidas en sesiones de webconferencia en línea con estudiantes, que también tenían una orientación fundamentalmente práctica con introducciones teóricas.

- Con la experiencia, y la demanda de los estudiantes de más ayuda para entender los contenidos teóricos del libro de texto, comencé un proyecto de elaboración de vídeos de una duración intermedia (15-20 min) con orientación puramente teórica a los que he llamado videoresúmenes. Como ya he dicho, este tipo de vídeos van dirigidos estudiantes del Grado en Matemáticas, y en particular tratan contenidos de Álgebra Lineal. 
Los video-resúmenes surgen motivados por una de las principales carencias detectadas en mi experiencia docente en la formación de los estudiantes que acceden en primer curso a estos estudios. Se trata de la falta de familiaridad con el uso formal del lenguaje lógico-matemático, que les hace muy difícil la lectura y comprensión de un libro de texto de matemáticas. Esto ocurre en todas las modalidades de enseñanza universitaria, pero cobra especial importancia en la enseñanza a distancia o semipresencial, en la que el libro es la herramienta principal del estudiante.

¿Cómo estudia Matemáticas un estudiante del Grado en Matemáticas? Es muy probable que la inmensa mayoría de estudiantes sin estudios universitarios previos, no hayan leído nunca un libro de matemáticas. Con frecuencia, los libros de matemáticas de Bachillerato se usan exclusivamente para apuntar los ejercicios que hay que hacer como tarea y mirar alguna fórmula. Aunque estos textos no presentan gran formalismo, ni siquiera está habituado el estudiante a la lectura de la exposición de los objetos matemáticos que va a estudiar y sus propiedades. Si el estudiante, como ocurre con relativa frecuencia en la UNED, tiene estudios preuniversitarios científicos o tecnológicos, tiene algo más de familiaridad con los procedimientos prácticos de la resolución de modelos de ejercicios (algunos de gran dificultad). Pero, en cualquiera de los dos casos, con formación previa o no, se han estudiado las Matemáticas como un conjunto de objetos (ecuaciones, funciones, matrices, etc.) y resultados (Teoremas, Proposiciones) que se usan como herramientas para resolver ciertos modelos de problemas más o menos estandarizados.

El estudiante del Grado en Matemáticas debe estudiar con un enfoque totalmente distinto al que pueda conocer previamente. Esta es la primera y gran dificultad para él, el salto cualitativo. Las Matemáticas son fundamentalmente teóricas y se expresan en un lenguaje formal muy estricto que cuesta ser leído por primera vez. El objetivo del matemático es llegar a comprender la naturaleza de los objetos matemáticos, los axiomas o propiedades que los definen y cómo a partir de estos axiomas se deducen (demuestran) otras propiedades nuevas que se enuncian como resultados que se podrán (después) aplicar posteriormente en diversos ámbitos. Por ejemplo, un estudiante, tras un curso de Álgebra Lineal, puede ver (o se le debe hacer ver) cómo todos los resultados que se exponen en su libro se han demostrado exclusivamente a partir de los ocho axiomas que definen la estructura de Espacio Vectorial.

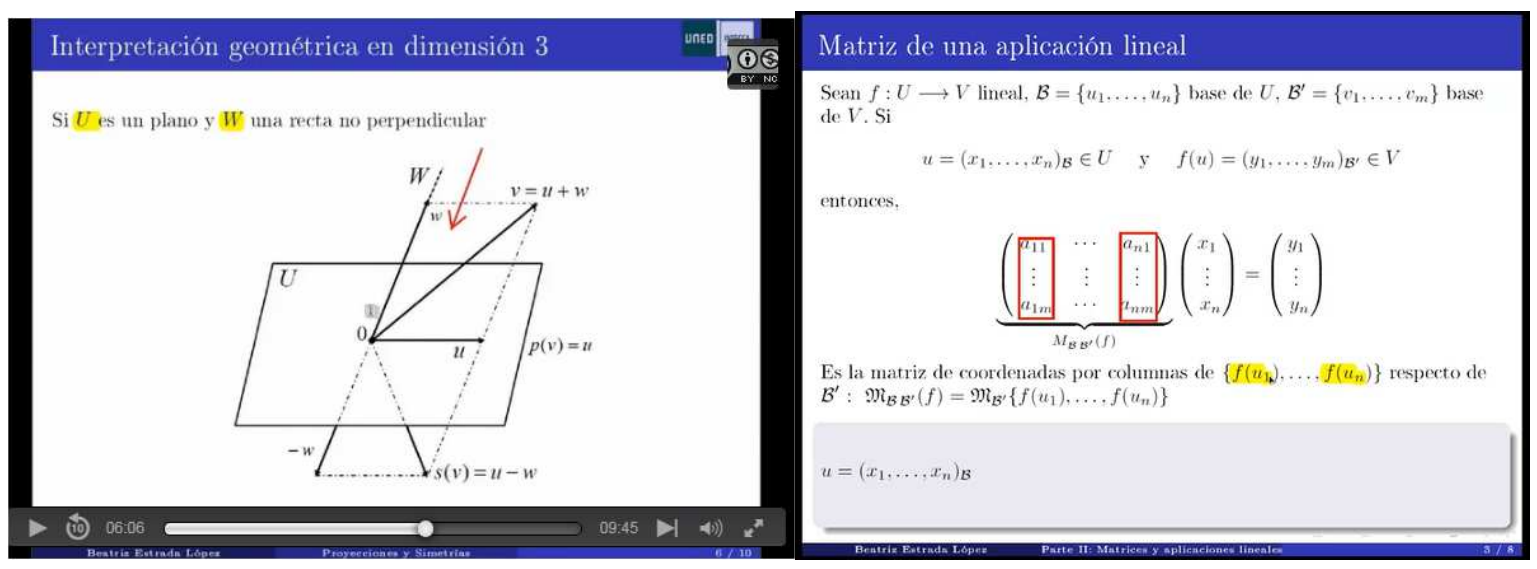

2 Capturas de imagen de dos video-resúmenes

Vídeo-resúmenes de Álgebra Lineal 
Teniendo como objetivo principal ayudar a familiarizar al estudiante de primer curso con la lectura del libro de texto, he realizado durante el curso 2015/16 un total de 7 video-resúmenes de entre 15 a 20 minutos de duración. En los vídeos se va haciendo una lectura de las definiciones de todos los objetos matemáticos y sus propiedades más importantes, que se enuncian en términos de Proposiciones y Teoremas, interrelacionándolos y ejemplificándolos. El uso por parte de los estudiantes es doble:

1.- Escuchar la lectura/exposición del profesor de forma resumida, les prepara antes del estudio.

2.- Tras el estudio, ayuda a interrelacionar y asentar conceptos. Se hace labor de síntesis.

Los vídeos han sido muy bien acogidos por parte de los estudiantes, que manifiestan explícitamente su utilidad, y también queda patente en el número de visualizaciones. Todos ellos están alojados en el repositorio Cadena Campus y se puede ver la evolución de su uso. En la siguiente tabla se recogen las visualizaciones tras haber terminado el curso de Álgebra Lineal en el primer semestre (datos de julio 2016).

\begin{tabular}{|l|c|c|}
\hline Video Resúmenes & Duración (min) & Visualizaciones \\
\hline \hline 1. Matrices. Parte I & $17: 05$ & 1911 \\
2. Matrices. Parte II & $13: 32$ & 1099 \\
3. Sistemas lineales & $13: 26$ & 569 \\
4. Espacios vectoriales & $18: 19$ & 845 \\
5. Subespacios vectoriales & $20: 40$ & 385 \\
6. Aplicaciones lineales. Parte I & $20: 18$ & 295 \\
7. Aplicaciones lineales. Parte II & $14: 59$ & 232 \\
\hline \hline & $\simeq 2$ horas & \\
\hline
\end{tabular}

El descenso en el número de visualizaciones se debe fundamentalmente a que los vídeos fueron apareciendo a lo largo del curso, según los fui elaborando. El primero de ellos fue el único que estuvo disponible prácticamente desde el inicio de curso, el último apenas dos semanas antes de los exámenes. Los resultados son de gran aceptación teniendo en cuenta el público al que iba dirigido (197 estudiantes se presentaron al examen, aunque había muchos más matriculados).

Tecnología. Para la elaboración de los video-resúmenes utilizo una presentación dinámica elaborada con el paquete beamer de LaTeX, el editor profesional de textos científicos, que genera un documento pdf. Un capturador de pantalla para la elaboración y posterior edición del vídeo (Camtasia) y un software adicional para hacer anotaciones a mano alzada o distintos tipos de marcas. Para esto último resulta de gran utilidad (aunque no es imprescindible) disponer de un dispositivo con pantalla táctil.

\section{Una reflexión final: ¿Videos largos o cortos?}

Existe la teoría de que un vídeo de duración superior a 5 o 6 minutos hace que se pierda la atención por el contenido y se recomienda la elaboración de las conocidas como "píldoras" o mini-vídeos. No es esa la experiencia que yo he tenido en la docencia universitaria en el Grado en Matemáticas. He podido comparar las visualizaciones de un video-resumen de $15 \mathrm{~min}$. con una sesión de webconferencia de 50 min. de duración en la que se explican parte de los contenidos del video-resumen y se hacen ejercicios. El número de visualizaciones del vídeo largo era superior al corto. 
La longitud, a mi modo de ver, debe ir en consonancia con el objetivo del vídeo. Si se quiere transmitir algo similar a lo que hace un profesor en un aula, entonces es necesario utilizar un formato largo, que tiene interés para el estudiante cuando está completamente enfocado en el material que él utiliza para preparar su asignatura. Si sólo se pretende resolver un ejercicio sencillo se puede utilizar el formato mini-vídeo. Mientras que los resúmenes de conceptos serían de una duración intermedia.

Los tres formatos tienen intereses distintos y complementarios. Todos resultan útiles para el estudiante si los contenidos que se presentan son adecuados, y especialmente si el autor es su propio profesor.

Beatriz Estrada López

Departamento de Matemáticas Fundamentales (UNED)

bestra@mat.uned.es 